Gut, 1974, 15, 112-115

\title{
Extraction of circulating endogenous gastrin by the gastric fundus
}

\author{
JOHN C. W. EVANS ${ }^{1}$, DAVID D. REEDER, HORST D. BECKER ${ }^{2}$, AND \\ JAMES C. THOMPSON \\ From the Department of Surgery, The University of Texas Medical Branch, Galveston, Texas
}

SUMMARY The effect of circulatory transit of the gastric fundus on serum levels of endogenous gastrin measured directly by radioimmunoassay has been studied in 14 dogs. Gastrin was measured in samples obtained simultaneously from the arterial inflow and venous outflow of the gastric fundus. During basal conditions, transit of the gastric fundus resulted in no change in gastrin concentration. During periods of stimulated gastrin release from the antrum, nine of the 14 dogs demonstrated a significant gastric acid secretory response. In these dogs there was a significant arteriovenous difference (approximately 30\%) in circulating gastrin values. In the remaining five dogs, which did not demonstrate a significant gastric acid secretory response, there was no arteriovenous difference in circulating gastrin values. It is concluded that the gastric fundus is an important site for the inactivation of stimulated levels of circulating gastrin.

The steady release of gastrin from the gastric antrum during basal conditions is greatly enhanced during periods of antral stimulation (Jackson, Reeder, and Thompson, 1972). The eventual fate of gastrin remains obscure, but it is clear that the immunochemical and physiological actions of gastrin are rapidly dissipated; exogenously administered heptadecapeptide gastrin (Reeder, Jackson, Brandt, and Thompson, 1972) or endogenous gastrin (Yalow and Berson, 1970) has a half-life of less than 10 minutes. The mechanisms and organs responsible for this rapid catabolism have yet to be accurately identified. Nephrectomy prolongs the half-life of exogenous gastrin (Clendinnen, Reeder, Brandt, and Thompson, 1973) and transit of either the kidney (Booth, Reeder, Hjelmquist, Brandt, and Thompson, 1973) or small bowel (Becker, Evans, Reeder, and Thompson, 1973) results in a decrease in circulating levels of endogenous gastrin, whereas transit of the liver has no effect (Reeder, Brandt, Watson, Hjelmquist, and Thompson, 1972).

Although the actions of gastrin are widespread (Thompson, 1969), the major target organ of the

${ }^{1}$ Present address: Department of Surgery, Royal Infirmary, Bristol, England.

Present address: Klinik und Poliklinik für Allgemeinchirurgie, 34, Gottingen, Gosslerstrasse 10, Germany.

Correspondence to: James C. Thompson, MD, Department of Surgery, The University of Texas Medical Branch, Galveston, Texas 77550.

Received for publication 29 October 1973. hormone is the gastric fundus. The present study was undertaken to determine the effect of circulatory transit of the gastric fundus on physiological levels of endogenous gastrin.

\section{Materials and Methods}

Experiments were conducted on 14 healthy, adult mongrel dogs weighing 16-19 kg. Each dog was fasted for 24 hours before the study and was anaesthetized preoperatively with intravenous pentobarbital sodium. A catheter for sampling blood was introduced into the right femoral artery. The serum gastrin concentration of blood samples obtained from this catheter was assumed to be the same as that in the arterial supply of the gastric fundus.

At laparotomy, the stomach was gently displayed and one of the veins draining the gastric fundus was carefully isolated proximal to its union with the splenic vein. A catheter for blood sampling was introduced into this vein; patency was maintained by intermittent irrigation with normal saline. The stomach was divided intoantral and fundic pouches by means of clamps placed at the antrofundic junction. A double-lumen catheter to allow perfusion of the antral pouch was placed into the antrum via a small duodenal incision and the pylorus was ligated around the catheter. A sump-type orogastric tube was placed in the fundus, and gastric secretions were collected at 30-minute intervals throughout the 
study. Acid output was measured by titration with $0 \cdot 1 \mathrm{~N} \mathrm{NaCl}$ with phenol red used as an indicator.

After collecting basal fundic secretions for a 30-minute period and basal blood samples from both arterial and venous catheters, the antrum was irrigated continuously with a $0.5 \%$ acetylcholine solution ( $\mathrm{pH} \mathrm{7.0)}$ ) for 90 minutes. At the end of this period, the antral pouch was drained, and irrigation was continued with $0 \cdot 1 \mathrm{~N} \mathrm{HCl}$ for another 60 minutes. Blood samples for serum gastrin determination were obtained from the arterial and venous catheters at 15 -minute intervals throughout the study.

Serum gastrin concentrations were determined by radioimmunoassay using a double-antibody technique which has been described previously (Jackson et al, 1972). The antigastrin antibody was developed in rabbits to synthetic human gastrin I (amino acid residues 2-17) conjugated to bovine serum albumin. All samples were assayed in duplicate and the reproducibility was within $10 \%$. The average of the duplicates was used as the serum concentration.

The results are expressed as serum concentrations of gastrin at any given time or as the integrated gastrin response over a given period (Thompson, Reeder, Bunchman, Becker, and Brandt, 1972). The integrated gastrin responses were calculated in the following manner: the average basal serum gastrin concentration was multiplied by the 75minute time period of antral stimulation (15-90 minutes after initiating stimulation) to obtain the basal integrated gastrin concentration. The stimulated integrated gastrin for each dog was calculated by subtracting the basal integrated gastrin concentration from the product of the 75 -minute time period multiplied by the average of the five serum gastrin concentrations measured during this period. The individual integrated gastrin responses for each dog were averaged and are expressed in picogramminutes per millilitre (pg-min/ml). A similar method was used to calculate the integrated gastrin response for the 45-minute period of antral acidification (105-150 minutes after initiating stimulation).

Results are expressed as the mean \pm 1 standard error. The Student $t$ test was used to analyse the data for statistical significance of differences between means. Differences with a P value of less than 0.05 were considered significant.

\section{Results}

Under basal conditions there was no significant difference between the concentration of gastrin in the arterial serum, $108 \pm 15$ picograms $(\mathrm{pg}) / \mathrm{ml}$ and in the venous serum, $99 \pm 10 \mathrm{pg} / \mathrm{ml}$ (fig 1). During antral stimulation with acetylcholine, gastrin

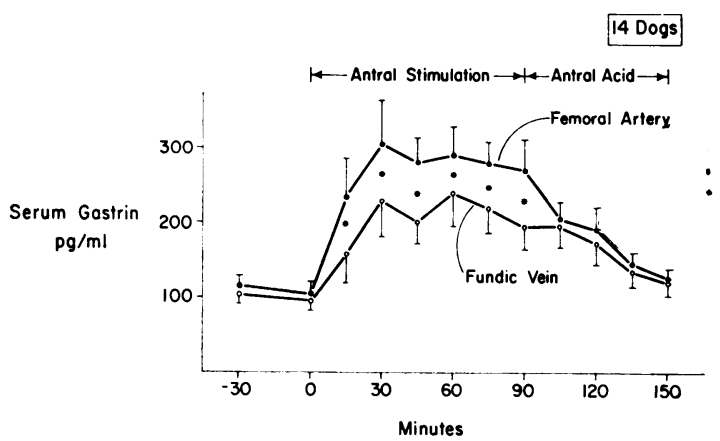

Fig 1 Concentrations of gastrin in the arterial inflow and venous outflow of the gastric fundus during periods of antral stimulation with acetylcholine and antral inhibition with acid. Asterisk indicates significant arteriovenous difference in concentration $(p<0.05)$. Narrow vertical bars in this and subsequent figures indicate 1 SEM.

levels increased in both arterial and venous samples, but concentrations were significantly lower in all of the venous samples compared to the arterial samples. The average arterial concentration of serum gastrin increased to $302 \pm 57 \mathrm{pg} / \mathrm{ml}$ at 30 minutes; the venous concentration at the same time was $221 \pm 46$ $\mathrm{pg} / \mathrm{ml}(\mathrm{P}<0 \cdot 05)$. Following the introduction of acid into the antral pouch, both arterial and venous gastrin values decreased to near basal. There was no significant arteriovenous difference at any time after the antrum was acidified.

In nine of the 14 dogs, there was a significant increase in gastric acid secretion (over basal) during irrigation of the antrum with acetylcholine (fig 2). Acid production increased from a basal value of $0.10 \pm 0.05 \mathrm{~m}$-equiv $/ 30$ minutes to a maximum value of $0.93 \pm 0.17 \mathrm{~m}$-equiv/30 minutes

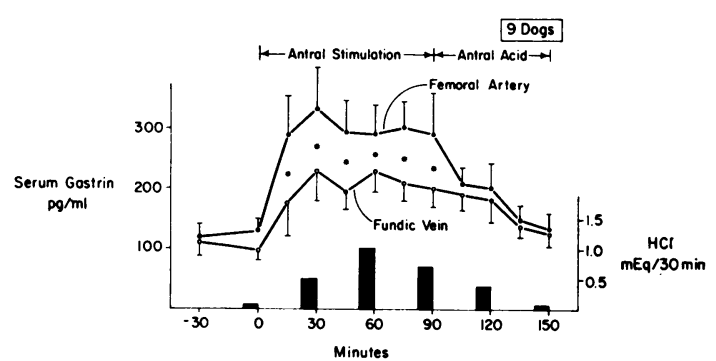

Fig 2 Concentration of gastrin in the arterial inflow and venous outflow of the gastric fundus during periods of antral stimulation with acetylcholine and antral inhibition with acid. Asterisks indicate a significant arteriovenous difference in concentration $(p<0.05)$. Included are the nine out of 14 dogs which demonstrated an increased acid output compared to basal. The bar graph represents acid output. 


\begin{tabular}{|c|c|c|c|c|}
\hline & \multicolumn{2}{|c|}{$\begin{array}{l}\text { Antral Stimulation } \\
(0 \cdot 1 \% \text { Acetylcholine })\end{array}$} & \multicolumn{2}{|c|}{$\begin{array}{l}\text { Antral Acidification } \\
(0 \cdot 1 \mathrm{NHCl})\end{array}$} \\
\hline & Femoral Artery & Fundic Vein & Femoral Artery & Fundic Vein \\
\hline $\begin{array}{l}\text { All dogs (14) } \\
\text { Secreting dogs (9) } \\
\text { Non-secreting dogs (5) }\end{array}$ & $\begin{array}{l}13137 \pm 2625 \\
13593 \pm 3853 \\
12318 \pm 3003\end{array}$ & $\begin{array}{l}8650^{1} \pm 1726 \\
7896^{1} \pm 1999 \\
9997 \pm 3465\end{array}$ & $\begin{array}{l}2934 \pm 696 \\
2692 \pm 896 \\
3370 \pm 1202\end{array}$ & $\begin{array}{l}2408 \pm 674 \\
2254 \pm 769 \\
2685 \pm 1407\end{array}$ \\
\hline
\end{tabular}

Table Integrated gastrin responses $(\mathrm{pg}-\mathrm{min} / \mathrm{ml})$ in femoral artery and fundic vein during periods of antral stimulation and antral acidification.

${ }^{1}$ Indicates venous values which are significantly lower than arterial values $(\mathrm{P}<0.05)$.

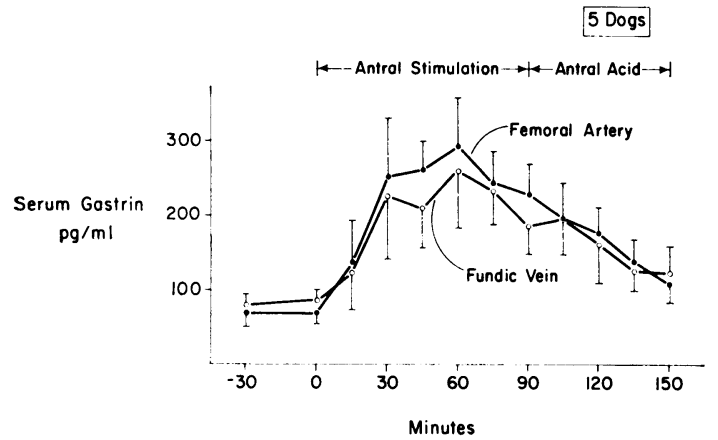

Fig 3 Concentration of gastrin in the arterial inflow and venous outflow of the gastric fundus during periods of antral stimulation with acetylcholine and antral inhibition with acid. Included are the five out of 14 dogs which demonstrated no acid secretory response.

$(P<0.05)$. Secretion decreased to basal levels after the introduction of acid into the antral pouch. In these nine dogs there was a significant difference between arterial and venous concentrations at all times during antral stimulation $(P<0.05)$, but no significant difference at any time after acidification of the antrum.

In the remaining five dogs there was no gastric secretory response to antral stimulation with acetylcholine, despite significant elevation of circulating arterial serum gastrin concentrations (fig 3). In these dogs there was no significant arteriovenous difference in gastrin concentrations at any time during the study.

The integrated gastrin levels for the periods of antral stimulation and antral acidification are shown in the table. There was no significant difference in arterial integrated gastrin output between the nine dogs which secreted acid and the five dogs which did not $(P>0.05)$. During antral stimulation there was a significant difference between arterial and venous levels $(P<0.05)$ in only those dogs that exhibited a significant gastric secretory output. No dogs showed a significant difference between arterial and venous integrated gastrin response levels during antral acidification.

\section{Discussion}

This study demonstrates that circulatory transit of the gastric fundus has no effect on serum gastrin concentration during basal conditions. Irrigation of the antrum with acetylcholine produced a notable elevation of circulating serum gastrin levels. In nine out of 14 dogs this elevation was accompanied by a significant increase in gastric acid secretion. The subsequent introduction of acid into the antrum abolished both the stimulated release of gastrin and the gastric secretory response. During the period of stimulated gastrin release and acid production, there was a significant difference between serum gastrin levels in the femoral artery, taken to represent the concentration in the fundic arterial supply, and the levels in the fundic vein.

The observation that the gastric fundus has no effect on basal concentrations of gastrin is similar to that found in studies on the kidney (Booth et al, 1973) and small bowel (Becker et al, 1973), which also have an effect only on stimulated levels of circulating endogenous gastrin. These findings suggest a threshold for activation of catabolic mechanisms for gastrin.

The loss of stimulated levels of gastrin on transit of the kidney, small bowel, or gastric fundus does not represent nonspecific uptake by tissue. Neither transit of the liver (Reeder et al, 1972) nor hind limb nor lung (unpublished studies) resulted in arteriovenous differences of basal or stimulated levels of gastrin.

Since gastrin in the present work was inactivated on transit of only the secreting gastric fundus, it is tempting to speculate that gastrin is fixed or taken up or actually degraded by the secreting parietal cell. Similar losses of gastrin, however, occur on transit of the kidney (Booth et al, 1973) and small bowel (Becker et al, 1973).

In five dogs there was no increase in gastric acid output despite elevations of circulating serum gastrin. Absence of acid secretion in these dogs was not due to lesser changes in gastrin as compared with the nine secreting dogs, since integrated gastrin 
output was actually slightly, although not significantly, higher. The failure of gastrin to stimulate acid secretion, a frequent finding under these conditions in anaesthetized dogs, was associated with no significant diminution in the serum gastrin concentration across the gastric fundus.

The failure of five dogs to secrete acid despite elevated levels of circulating gastrin must be attributed to the effect of anaesthesia on the parietal cell. Many investigators have reported a suppression of gastric secretion by anaesthesia (Merendino, 1948;Skyring, Milton, and Maxwell, 1961 ; Powell and Hirschowitz, 1967; Evans, Reeder, and Thompson, 1972). Altered cell function during anaesthesia may also explain the absence of any difference in gastrin concentration across the gastric fundus in dogs that had no gastric secretory response to elevated levels of circulating serum gastrin.

We conclude that circulatory transit of the gastric fundus produces a significant reduction in stimulated serum concentrations of gastrin as determined by radioimmunoassay.

Supported by grant AM 15241 from The National Institutes of Health and by a grant from The John A. Hartford Foundation, Inc.
References

Becker, H. D., Reeder, D. D., and Thompson, J. C. (1973). Extraction of circulating endogenous gastrin by the small bowel. Gastroenterology, 65, 903-906.

Booth, R. A. D., Reeder, D. D., Hjelmquist, U. B., Brandt, E. N., Jr., and Thompson, J. C. (1973). Renal inactivation of endogenous gastrin in dogs. Arch. Surg., 106, 851-854.

Clendinnen, B. G., Reeder, D. D., Brandt, E. N., Jr., and Thompson, J. C. (1973). Effect of nephrectomy on the rate and pattern of disappearance of exogenous gastrin in dogs. Gut, 14, 462-467.

Evans, J. C. W., Reeder, D. D., and Thompson, J. C. (1972). Effect of anesthesia on gastric secretion and circulating gastrin. Physiologist, 15, 129.

Jackson, B. M., Reeder, D. D., and Thompson, J. C. (1972). Dynamic characteristics of gastrin release. Amer. J. Surg., 123, 137-142.

Merendino, K. A. (1948). The effect of phenobarbital on the gastric secretions in dog and man. Gastroenterology, 10, 531-539.

Powell, D. W., and Hirschowitz, B. I. (1967). Sodium pentobarbital depression of histamine- or insulin-stimulated gastric secretion. Amer. J. Physiol., 212, 1001-1006.

Reeder, D. D., Brandt, E. N., Jr., Watson, L. C., Hjelmquist, U. B. E., and Thompson, J. C. (1972). Pre- and posthepatic measurements of mass of endogenous gastrin. Surgery, 72, 34-41.

Reeder, D. D., Jackson, B. M., Brandt, E. N., Jr., and Thompson. J. C. (1972). Rate and pattern of disappearance of exogenous gastrin in dogs. Amer. J. Physiol., 222, 1571-1574.

Skyring, A. P., Milton, G. W., and Maxwell, G. A. (1961). Effect of sodium pentobarbital on histamine stimulated gastric secretion, Amer. J. Physiol., 201, 574-576.

Thompson, J. C. (1969). Gastrin and gastric secretion. Ann. Rev. Med., 20, 291-314.

Thompson, J. C., Reeder, D. D., Bunchman, H. H., Becker, H. D., and Brandt, E. N., Jr. (1972). Effect of secretin on circulating gastrin. Ann. Surg., 176, 384-393.

Yalow, R. S., and Berson, S. A. (1970). Radioimmunoassay of gastrin. Gastroenterology, 58, 1-14. 